\title{
EXPLORING THE POTENTIALS OF ARTIFICIAL INTELLGIENCE IN THE JUDICIARY
}

\author{
Oloruntoba Samson Abiodun \\ Department of Computer Science \\ Federal Polytechnic, Ilaro, Nigeria
}

\author{
Akinode John Lekan \\ Department of Computer Science \\ Federal Polytechnic, Ilaro, Nigeria
}

\begin{abstract}
In recent years, there has been massive progress in Artificial Intelligence (AI) with the development of machine learning, deep neural networks, natural language processing, computer vision and robotics. These techniques are now actively being applied in the judiciary with many of the legal service activities currently being delivered by lawyers predicted to be taken over by AI in the coming years. This paper explores the potentials and efficiency of Artificial intelligence (AI) in justice delivery. The paper has two objectives: first to highlight the main applications of $\mathrm{AI}$ in justice administrations through some examples of AI tools recently developed; second, to assess the ethical challenges of AI in the judiciary. Artificial Intelligence algorithms are starting to support lawyers, for instance, through artificial intelligence search tools, or to support justice administrations with predictive technologies and business analytics based on the computation of Big Data. Using the concept of Artificial Intelligence (AI), Legal knowledgebased tools may accelerate the service delivery of legal professionals from typical searching of related case journals to extraction of precise information in a customized manner.
\end{abstract}

Keywords-Artificial Intelligence, Judiciary, Justice Administration, Algorithms, Ethical Challenges

\section{INTRODUCTION}

The current socio-economic development stage is characterized by digital transformations, which has impacted a great section of the economy and businesses. The domain of legal services is not an exception. The effect of technology on the legal sector is not new, the development of the internet, emails and legal databases online have been around for a long time (Jie-jing YAO and Peng Hui ,2020). The activities of a present-day lawyer are linked with the active use of information technology tools that allow information search for necessary regulatory and legal material, law enforcement practice, quickly information retrieval, and effective informed decisions. These processes hinged on the use of automated information data systems, these include computer and communication equipment, software, linguistic tools and information resources designed to process and provide information for decision-making purposes. Most of the automated information data systems operate on the basis of artificial intelligence technology (Deltsova N.V. ,2021).

Artificial Intelligence (AI) is attracting an enormous amount of attention in the media and public discourse. Wellpublicized recent successes for AI included self-driving cars and self-teaching board game champions. Artificial intelligence is having an impact on our social lives. Its effect also extends to the process of law-making and the application of law (Gabriele Buchholtz,2020). The expanding use of Artificial Intelligence and the World Wide Web has led to the development and use of Online Dispute Resolution (John Zeleznikow ,2017). Artificial Intelligence has a high capability to improve accuracy, predictability and efficiency of the legal sector with precision and speed unmatchable by humans. According to Teng $\mathrm{Hu}$ and Huafeng Lu. (2019), AI will determine the future of professional development and talent training in law science education. The combination of A.I. Systems and Machine Learning are not a futurist idea, there are already different technologies integrated into the operation of the judiciary around the globe, like recidivism prediction in the criminal context, predictive justice, and many others.

Digitalization of the judicial system has happened already around the globe in many diverse ways, however, the automation of the judicial system via Artificial Intelligence and Machine Learning is still being evaluated and still in early stages. Major countries like Canada, United Kingdom, Ireland and even the European Court of Justice have initiated some level of digitalization like e-filing, e-conferencing and prediction systems (Paula C. Arias ,2020). However, China is taking the lead on automatization of the judicial system, in fact, it has already established a few internet courts in the Hanzhou, Beijing and Guangzhou cities. The courts operate based on big data, blockchain technology and artificial intelligence.

The introduction of different software tools is beginning to have a considerable impact on different aspects of lawyers' work, including those tasks that depend upon expert human judgment, such as predicting court outcomes. The emergence of these new software tools presents new challenges and new opportunities. For instance, in 2016, Ross, the first AI lawyer 


\section{International Journal of Engineering Applied Sciences and Technology, 2020 \\ Vol. 5, Issue 8, ISSN No. 2455-2143, Pages 23-27 \\ Published Online December 2020 in IJEAST (http://www.ijeast.com)}

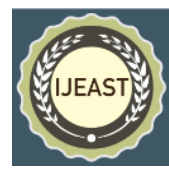

in the history supported by Watson, IBM's cognitive computer, was "employed" by an American law firm. The AI tool allows communication with lawyers in human language, give people an experience of working with prospective employees, and be able to deal with certain bankruptcy consulting issues (Liberatore S.,2018). Similarly, DoNotPay, a robot lawyer, which can help users to challenge traffic tickets and to prepare legal documents was developed in the UK. McGinnis and Russell Pearce (2014) reiterated that machine intelligence will cause a "great disruption" in the market for legal services. The authors established that five major areas of legal services will be affected: legal search, discovery, brief generation, document generation, brief generation, and prediction of case. However, the introduction of Artificial intelligence (AI) raises new challenges for justice and judicial systems. In light of these facts, using algorithms and predictive methods to make decisions especially ones that may affect the freedom of individuals requires further study and attention.

This paper has two objectives: first to highlight the main applications of AI in justice administrations through some examples of AI tools recently developed; second, to assess the ethical challenges of AI in the judiciary. The rest of the paper is organized as follows. Proposed embedding and extraction algorithms are explained in section II. Experimental results are presented in section III. Concluding remarks are given in section IV.

\section{LITERATURE REVIEW}

\section{A. What is Artificial Intelligience -}

In this section, we briefly introduce specific concepts in artificial intelligence that will likely enhance the development of computational tools for judicial activities. With the excellent performance of the artificial intelligence systems like AlphaGo in recent years, artificial intelligence has become a hot topic among publics.

Artificial intelligence (AI) has been defined as a field of computer science dealing with the capability and simulation of a machine to imitate intelligent human behavior. Habeeb Ahmed (2017) describe AI as field of computer science that highlights the development of intelligent machines that work and behave like humans. The activities which artificial intelligence are designed for include: Speech recognition, Visual perception, translation between languages, Learning, Planning and Problem solving. Handel, L. M et al (2020) also describe AI as a branch of computer science that study the relation between computation and cognition. The purpose of $\mathrm{AI}$ is to perform tasks that normally require human intelligence. AI programs may mimic or simulate cognitive behaviors or traits associated with human intelligence such as reasoning, problem solving, and learning. AI applications are embedded in the infrastructure of many products and industries such as search engines, speech recognition, robot control, medical diagnoses, web search, advertising and even toys

\section{B. Subdomains of Artificial Intelligience:Methods and Application Areas -}

Handel, L. M et al (2020) highlighted some of the areas of AI, these include the following:

- Machine Learning -Machine Learning (ML) is one of the sub-domains of AI. ML provides the machines with the ability to learn automatically and advances from the past experience without the help of explicit programming. For example, decision tree learning and version space learning.

- Computer Vision - The computer vision (CV) system operates as vision sensor and provides high-level data about the surrounding. It also includes of pattern recognition and learning techniques. for example, object recognition and understanding of image.

- $\quad$ Speech Processing - It is the quality of a system or program to recognize words and phrases from verbal communication and translate them to a machinereadable layout. For example, speech recognition and production

- Natural Language Processing - A subfield of AI, it is mainly based on the connections of computers and natural languages. It is regarding the process of converting the programs to process and analyze huge amount of natural language data. For example, machine translation

- Planning - It is the process of decision making by machines or programs to perform a specific task. It is about selecting a succession of actions with a high possibility of completing it. For example, scheduling and game playing.

- Expert Systems - It is a computer-based system that imitates the decision-making capability of a human. These systems are planned to resolve multifaceted problems with the help of reasoning through bodies of knowledge, represented mainly as if-then rules rather than through conventional procedural code. For example, decision making and teaching systems.

- Evolutionary Computation - A sub-domain of AI and Soft Computing that comprises of algorithms for global optimization, Evolutionary Computation is a population-based trial and error problem solver that has characteristics of stochastic optimization. For example, genetic algorithms and programming.

- Robotics - These are the artificial agent that aims at influencing the objects by recognizing, selecting, shifting and demolishing it. For example, intelligent control and autonomous exploration.

- Neural Network - It is mainly characterized with the help of adaptive weights and paths connecting the 


\section{International Journal of Engineering Applied Sciences and Technology, 2020 \\ Vol. 5, Issue 8, ISSN No. 2455-2143, Pages 23-27 \\ Published Online December 2020 in IJEAST (http://www.ijeast.com)}

neurons that is able to tune by learning algorithm that gains knowledge from the observed data to have an advanced model. For example, brain modeling, classification and time series prediction.

- Deep learning - a class of learning procedures, has facilitated object recognition in images, video labeling, and activity recognition, and is making significant inroads into other areas of perception, such as audio, speech, and natural language processing.

\section{AI APPLICATIONS FOR JUDICIARY PRATICE}

Artificial Intelligence (AI) technology will profoundly change the operation mode of the legal industry itself. AI has shown promises in simplifying most tasks in the judiciary. These include: case push, knowledge index query, auxiliary conviction, sentencing reference, evidence review and judgment, integrated support in court, voice / image reading and transformation, case management, deviation analysis, judicial / administrative connection, automatic generation of documents and peer-to-peer proofreading (Handel L. M et al,2020).

\section{Predictive Justice}

Software anticipating a judicial decision based on the analysis of a large quantity of case law. Predictive systems are used mainly in pre-litigation planning. AI-driven software can examine huge numbers of cases and all the publicly available court documents and rulings made by judges in the past up to the present day that are relevant to a case along, with many other types of useful public data:

\section{Predictive policing algorithms}

Predictive policing algorithms leverage on historic crime data as their input, identify trends of interest, and make predictions on likely outcomes. Predictive software has been used by police to determine, for example, individuals that may have been involved in gun violence as either the perpetrator or the victim (Davey M,2016). Based on a certain number of factors such as an, employment, individual's age and criminal histories of family members, an algorithm calculates a numerical score and classifies individuals as low, medium, or high risk of recommitting in the future. Similar models have also been used to determine the risk of a defendant in committing crimes in the future and the likelihood of recidivism (Cheng, Kar Mun,2018).

Natural Language Processing (NLP) for Lawyers and Judges

Natural language processing examines the use words and phrases to draw connections within and across written or spoken language describe the ability of machines to understand and inteprete human language. Its application in the legal domain include linguistic analysis of legal texts, detection of arguments in legal texts.

\section{Intelligence, Surveillance, and Reconnaissance}

AI technology is expected to be useful in intelligence gathering due to the large data sets available for analysis. For instance, AI could integrate computer vision and machine learning algorithms into intelligence collection cells that would comb through footage from uninhabited aerial vehicles and automatically identify hostile activity for targeting. In this capacity, AI is intended to automate the work of human analysts who currently spend hours sifting through drone footage for actionable information, potentially freeing analysts to make more efficient and timely decisions based on the data.

\section{Judicial Analytics}

The term 'judicial analytics' describes the analysis of data (including judgments and other public records of the work of judges) using artificial intelligence (AI) and machine learning to monitor, understand or predict judicial behavior. Pamela Stewart and Anita Stuhmcke (2020). Judicial analytics is the use of data to monitor, understand and predict judicial behaviour.

\section{Document Automation.}

Some law firms are also beginning to adapt such technology by drafting documents through automated software. Many such software companies claim that the final document, which could take days by manual human drafting, is generated in a matter of minutes.

\section{MACHINE LEARNING TOOLS FOR JUDICIAR PRATICES}

The use of AI systems to support the work of legal practitioners has initially been observed in both public and private sectors. Anderson T et al(2020) and Riya Sil et al(2019) listed some of the major ML tools for judicial services. These include the following:

1)ROSS Intelligence in the U.S.A. It is created by the IBM legal research service for U.S. law and is powered by artificial intelligence. ROSS is based on the now famous Watson - a question-answering computer system capable of answering questions posed in natural language, developed in IBM's DeepQA project.

2)Predictive in France. It is a predictive analytics tool for estimating a success rate of court proceedings. Authors of Predictice claim that the system can analyse one million judicial decisions within 1 


\section{International Journal of Engineering Applied Sciences and Technology, 2020 \\ Vol. 5, Issue 8, ISSN No. 2455-2143, Pages 23-27 \\ Published Online December 2020 in IJEAST (http://www.ijeast.com)}

second, and in the last two years they started cooperation with over four hundred lawyers.

3)Luminance in the UK. It is a machine learning system which improves legal analytics by reading, understanding, and learning from analyzed documents. Luminance was launched in 2016, and since then it has been said to be used by over 14 of the global TOP 100 law fi rms. Its pattern recognition technology, advanced statistical probability analysis, supervised and unsupervised machine learning methods are said to allow identifying similarities, differences, and anomalies at all levels of the review of legal documents; thus, the system can be used in, e.g., due diligence or compliance analysis.

4)Victor in Brazil. This is an on-going project conceived by the Brazil Supreme Court in partnership with the University of Brasília. The tool is designed aims to support the Brazilian Supreme Court by providing analysis of the cases that reach the Court, using document analysis and natural language processing tools.

5)COMPASS: COMPAS Algorithm It is a risk assessment algorithm which is utilized for prediction of risk of committing another crime by the defendant. Using proprietary algorithm, it provides the types of advice that the convict requires which includes information related to the case that may be required during case sentencing.

6)eBrevia: In eBreviaAI is used along with Machine Learning and Natural Language Processing technology to extract relevant data from legal contracts and other documents, bring exceptional accuracy and speed to due diligence and lease abstraction to guide lawyers for analysis.

7)CASEIQ: The CaseIQ software is virtual legal research assistant that automatically analyzes the language of the brief and feeds the information into a complex predictive algorithm to highlight potential missing points of law, or alternative arguments

\section{ETHICAL CHALLENGES ON THE IMPLEMENTATION OF AI IN THE JUDICIAR}

This section explored the admissibility of using artificial intelligence (AI) tools in judiciary, and consider the ethical aspects of AI-application in judicial proceedings. Ethical issues surrounding AI use in law often share a common theme. As AI becomes increasingly integrated within the legal system, how can society ensure that core legal values are preserved? The use of $\mathrm{AI}$ raises more questions than resolves our concerns with the administration of justice.

\section{Dataset Biases}

Data biases can subtly creep into AI predictive models, and this can be hard to detect. In prediction techniques in AI, some of the choices may include the type of datasets to use, what data to exclude or include, what AI models to use or AI Algorithms to select, what information to highlight or deemphasize. The totality of these subtle choices might result in more or less favorable treatment to various social groups. In the light of this, the use of such AI systems in decision-making may subtly shift social and political power dynamics by providing more favorable automated decisions for certain groups over others.

\section{Unequal treatment from AI-based decisions}

Equal treatment for all under the law, regardless of status, is a core value in most legal systems. This norm posits that legal decisions should be based upon the law and the facts, but not upon a party's socio-economic, political, racial, ethic, gender background or a variety of other individual characteristics that are illegal or inappropriate to consider. Defendants in the same circumstances should be treated the same under the law regardless of status. The use of AI systems by judges (or other legal officials) to make decisions has raised concerns about this Unequal treatment from AI-based decisions can occur for a few reasons. Sometimes this happens when there are existing structural inequalities in society and these inequalities become reflected in the data used by AI-systems. For example, let's imagine that the risk-prediction system discussed earlier was based partly upon historical police arrest data. In that example, the AI algorithm discovered a feature that was predictive of reoffence: a defendant's history of having re-offended after a past encounter with the justice system was highly indicative of a future offense upon release.

\section{CONCLUSION}

In this paper, we have explored the potentials and risks of artificial intelligence in the judiciary based on the current state of knowledge. AI has proven itself to be useful in the judiciary. However, there is no evidence that robots are going to carry out the work of a judge. A lot of work still needed to be done before AI can thrive in Court.Explanability, a crucial component of trustworthy AI is still not feasible. As the legal information is becoming sophisticated, Artificial intelligence can also assist with suggestions and advice. The central ethical challenge is to identify the way in which the use of AI may be shifting core legal values, and to ensure that these crucial values are preserved in the technological transition. While it appears to post threat to some core legal businesses, the 


\section{International Journal of Engineering Applied Sciences and Technology, 2020 \\ Vol. 5, Issue 8, ISSN No. 2455-2143, Pages 23-27 \\ Published Online December 2020 in IJEAST (http://www.ijeast.com)}

overwhelming development of AI in legal practice cannot be overemphasized.

\section{REFERENCE}

[1] Anderson T., Torreggiani W.C., Munk P.L., Mallinson P.I. (2020) The impact of the introduction of artificial intelligence in radiology and its potential legal implications in the UK and Ireland. BJR Open 2020; 2: 20200030.

[2] Cheng, Kar Mun. (2018). Predictive Analytics in the Criminal Justice System: Media Depictions and Framing. Honors Program Theses. 62. https://scholarship.rollins.edu/honors/62

[3] Christopher Bishop. (2018). Pattern Recognition and Machine Learning, Springer Verlag.

[4] Davey M. (2016). Chicago police try to predict who may shoot or be shot.https://www.nytimes.com/2016/05/24/us/armed-with-datachicago-police-try-to-predict-whomay-shoot-or-be-shot.html.

[5] Deltsova N.V. (2021) Artificial Intelligence Technologies in the Field of Legal Services: Relevant Aspects. In: Ashmarina S., Mantulenko V. (eds) Current Achievements, Challenges and Digital Chances of Knowledge Based Economy. Lecture Notes in Networks and Systems, vol 133. Springer, Cham. https://doi.org/10.1007/978-3-030-474584_47

[6] Gabriele Buchholtz (2020). Challenges to the Rule of Law ISSN 2367-3370 ISSN 2367-3389 (electronic) Lecture Notes in Networks and Systems ISBN 978-3-030-47457-7 ISBN 9783-030-47458-4 (eBook) https://doi.org/10.1007/978-3-03047458-4@ Springer Nature Switzerland AG 2021

[7] Handel, L. M., Gaudette, L. W., Fleming, M. R., \& Arnold, R. A. (2020). Artificial Intelligence in Higher Education at Worcester Polytechnic Institute. Retrieved from https://digitalcommons.wpi.edu/iqp-all/5784

[8] Habeeb, A. (2017). Artificial intelligence Ahmed Habeeb University of Mansoura. Research Gate, 7(2). https://doi.org/10.13140/RG.2.2.25350.88645

[9] John Zeleznikow. (2017). Can Artificial intelligience and Online Dispute Enhance Efficiency and Effectiveness in Courts. International Journal of Court Administration.Vol 8.No.2.ISSN 2156-7964

[10] Jie-jing YAO and Peng Hui .(2020). Research on The Application of Artificial Intelligence in Judicial Trial: Experience from China.J. Phys.: Conf. Ser. 1487012013

[11] Liberatore S.(2016). Your AI Lawyer will See You Now: IBM's ROSS Becomes World's First .Artificially Intelligent Attorney [EB/OL].(2016-05-16)[2018-04-07].
[12] McGinnis and Russell Pearce(2014) The Great Disruption: How Machine Intelligence Will Transform the Role of Lawyers in the Delivery of Legal Services, In On New Legal Products \& Services, Reading List, Resources

[13] Paula C. Arias.(2020). Artificial Intelligence \& Machine Learning: a model for a new judicial system. Revista Internacional Jurídica y Empresarial. Número 3. Año 2020

[14] Surden, Harry. (2019). The Ethics of Artificial Intelligence in Law: Basic Questions.Forthcoming chapter in Oxford Handbook of Ethics of AI, 2020, U of Colorado Law Legal Studies Research Paper No. 19-29, Available at SSRN: https://ssrn.com/abstract=3441303

[15] Teng Hu and Huafeng Lu. (2019). Study on the Influence of Artificial Intelligence on Legal Profession. Advances in Economics, Business and Management Research, volume 110 5th International Conference on Economics, Management, Law and Education (EMLE 2019) 